\title{
Otimização de Múltiplos Objetivos na Soldagem de Revestimento de Chapas de Aço Carbono ABNT 1020 Utilizando Arame Tubular Inoxidável Austenítico
}

\section{(Multi-Objective Optimization in Cladding of ABNT 1020 Carbon Steel Plate Using an Austenitic Stainless Steel Cored Wire)}

\author{
José Henrique de Freitas Gomes ${ }^{1}$, Sebastião Carlos da Costa ${ }^{1}$, Anderson Paulo de Paival, Pedro Paulo Balestrassi ${ }^{1}$ \\ ${ }^{1}$ Universidade Federal de Itajubá, Instituto de Engenharia de Produção e Gestão, Itajubá, MG, Brasil, ze_henriquefg@yahoo.com.br
}

\begin{abstract}
Resumo
A soldagem de revestimento de aços carbono com aços inoxidáveis tem obtido destaque no meio industrial por permitir que superfícies anti-corrosivas sejam obtidas a partir de materiais de baixo custo. No entanto, visando garantir a qualidade final dos revestimentos, é importante que o procedimento de soldagem seja bem ajustado, para que os cordões sejam depositados com a geometria desejada, com produtividade e sem defeitos. O objetivo deste trabalho é a otimização de múltiplas características do processo de revestimento de chapas de aço carbono ABNT 1020 utilizando arame tubular de aço inoxidável ABNT 316L. As características otimizadas incluem a largura, penetração, reforço e diluição, que representam a geometria do cordão. A produtividade foi maximizada através da taxa de deposição e do rendimento do processo. Como respostas de qualidade, considerou-se a formação de escória e o aspecto superficial. A estratégia de modelagem e otimização foi baseada em uma combinação da Metodologia de Superfície de Resposta, Método do Critério Global e Algoritmo Genético. Os resultados indicam que os modelos de superfície de resposta desenvolvidos para as características do processo apresentaram altos ajustes. O Método do Critério Global e o Algoritmo Genético foram aplicados com sucesso, o que permitiu a identificação do ponto de ótimo.
\end{abstract}

Palavras-chave: Otimização multi-objetiva; Soldagem de revestimento; Metodologia de Superfície de Resposta; Método do Critério Global.

Abstract: The stainless steel cladding process has highlighted in the industrial environment for allowing anti-corrosive surfaces are made from low cost materials. However, in order to ensure the claddings final quality, it is important that the welding procedure is well adjusted, so that the welds are deposited with the desired geometry, with productivity and flawless. The aim of this work is the multiple characteristics optimization in cladding of ABNT 1020 carbon steel plates using ABNT 316L stainless steel cored wire. The optimized features include the bead width, penetration, reinforcement and dilution, representing the weld bead geometry. Productivity has been maximized through the deposition rate and process yield. As quality responses, we considered the slag formation and surface appearance. The modeling and optimization strategy was based on a combination of Response Surface Methodology, Global Criterion Method and Genetic Algorithm. The results indicate that the response surface models developed for the process characteristics had high adjustments. The Global Criterion Method and Genetic Algorithm were implemented successfully, allowing the results optimization.

Key-words: Multi-objective optimization; Cladding; Response Surface Methodology; Global Criterion Method.

\section{Introdução}

A soldagem de revestimento é o processo em que uma camada de metal de adição é depositada sobre a superfície de outro material com o objetivo de obter propriedades ou dimensões desejadas. É geralmente empregada para prorrogar a vida útil de peças que não possuem todas as propriedades necessárias para uma dada aplicação, para recuperação de elementos afetados pelo desgaste ou corrosão ou para criação de superfícies com características especiais [1,2].

Das aplicações anteriores, Murugan e Parmar [3] afirmam que o emprego da soldagem de revestimento para a criação de

(Recebido em 03/01/2011; Texto final em 29/08/2011). superfícies com características especiais tem aumentado no ambiente industrial e, considerando os diversos tipos de materiais para revestimentos, os aços inoxidáveis se caracterizam como uma das aplicações mais frequentes. Para estes casos, aços inoxidáveis são geralmente depositados sobre superfícies de aços carbono ou aços de baixa liga, produzindo uma camada com propriedades anti-corrosivas e de resistência necessárias para suportar ambientes sujeitos ao alto desgaste por corrosão. Os resultados deste processo têm tornado essa aplicação bastante atrativa, na medida em que superfícies resistentes à ambientes corrosivos podem ser produzidas a partir de materiais comuns e de menor custo, em comparação ao emprego de componentes puramente de aços inoxidáveis, os quais apresentam custos elevados. Portanto, as aplicações da soldagem de revestimento de aços carbono com aços inoxidáveis se estendem entre os mais diversos tipos de indústrias, como as petrolíferas, químicas, alimentícias, agrícolas, nucleares, navais, ferroviária, de 

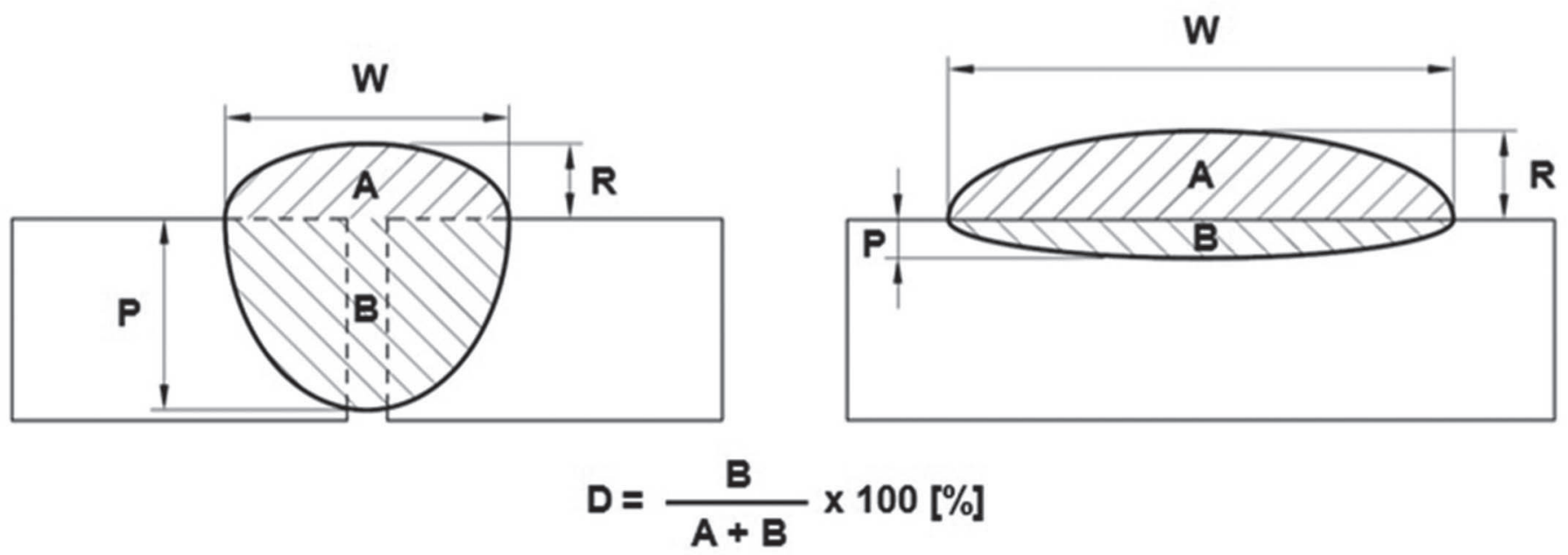

(a)

(b)

Figura 1 - Perfil geométrico desejado do cordão de solda: a) união da junta soldada (aplicações convencionais); b) soldagem de revestimento

construção civil, entre várias outras [4, 5].

A principal diferença da soldagem de revestimento em relação às aplicações convencionais de soldagem diz respeito à geometria do cordão de solda. Ao contrário das aplicações convencionais, em que é desejável alta penetração (P) para garantir a resistência da junta soldada (Figura 1a), na soldagem de revestimento o perfil geométrico desejado se resume a grandes larguras do cordão (W), altos reforços (R), baixas penetrações (P) e baixos percentuais de diluição (D) (Figura 1b) $[6,7]$. A obtenção deste perfil geométrico característico é importante para que o processo de revestimento permita recobrir a maior área possível com o menor número de passes, resultando em economias significativas de materiais e tempo.

Outro aspecto de grande importância para o processo de revestimento é o controle da diluição. Este controle, segundo vários pesquisadores, é uma das principais características para assegurar a qualidade final dos revestimentos [7-9]. Shahi e Pandey [10] argumentam que a composição e as propriedades dos componentes revestidos são fortemente influenciadas pela diluição. Para o processo de revestimento de aços carbono com aços inoxidáveis, o aumento da diluição reduz os elementos de liga e aumenta o conteúdo de carbono na camada revestida, reduzindo as propriedades de resistência à corrosão além de causar outros problemas metalúrgicos. Portanto, o estudo e o desenvolvimento de procedimentos capazes de oferecer uma diluição ótima tem se tornado muito importante.

No entanto, os resultados da soldagem de revestimento de aços carbono com aços inoxidáveis não se limitam apenas ao controle da diluição e à obtenção do perfil geométrico desejado. Tendo em vista que os ambientes industriais vêm fazendo uma exigência cada vez maior por processos capazes de combinar requisitos mínimos de qualidade com elevados índices de produtividade, a otimização deste processo também deve levar em consideração outras características. Nesse contexto, a produtividade deve ser otimizada para que a operação de soldagem permita a deposição de cordões com altas taxas de produção e máximo rendimento. Quanto à qualidade, é desejável que o cordão de revestimento ótimo seja isento de defeitos, ao mesmo tempo em que apresente um bom aspecto visual.

Diante de tais considerações, o presente trabalho tem como objetivo a otimização de múltiplas características da soldagem com arame tubular para o revestimento de chapas de aço carbono ABNT 1020 utilizando aço inoxidável ABNT 316L. Deseja-se identificar a combinação ótima dos parâmetros de soldagem que ofereçam os melhores resultados quanto à geometria do cordão de revestimento e à produtividade do processo, garantindo, ao mesmo tempo, níveis mínimos de qualidade superficial. A geometria do cordão foi representada através da largura, penetração, reforço e diluição. As respostas de produtividade incluíram a taxa de deposição e o rendimento do processo. Para a qualidade superficial, foram consideradas a formação de escória e o aspecto superficial. Os parâmetros de soldagem analisados compreendem a velocidade de alimentação do arame, tensão, velocidade de soldagem e distância bico de contato peça.

A escolha pelo emprego da soldagem com arame tubular se justifica pelo fato desta reunir algumas vantagens condizentes com os objetivos deste trabalho, como a obtenção de altas taxas de deposição, mínimo desperdício do eletrodo, flexibilidade do processo, alta qualidade da solda e excelente controle da poça de fusão [11].

A esquematização deste objeto de estudo, conforme apresentada pela Figura 2, caracteriza o processo em questão como um problema complexo de otimização, na medida em que estão definidas quatro variáveis de entrada e oito variáveis de resposta (processo multivariado e multi-objetivo). Dessa forma, a otimização individual dos múltiplos objetivos pode levar o problema à identificação de conflitos de interesse, já que a combinação dos parâmetros de entrada que oferece a melhor condição para uma dada resposta pode não ser a mesma que otimiza as demais. Por outro lado, os parâmetros de entrada não devem ser tratados de forma isolada, sob o risco de interações significativas entre eles existirem e serem negligenciadas. 


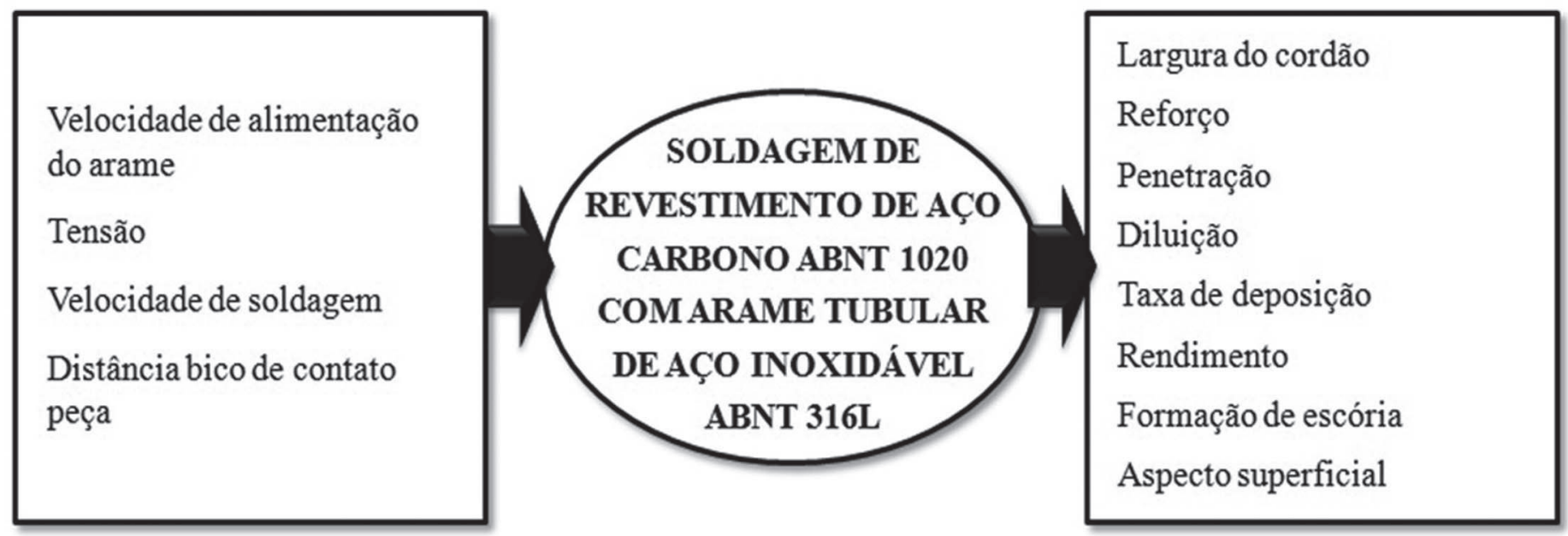

Figura 2 - Esquematização do objeto de estudo

Portanto, técnicas específicas para a otimização de múltiplos objetivos devem ser utilizadas, a fim de que as múltiplas respostas sejam otimizadas de forma simultânea e o problema convirja para uma solução global entre elas.

Entre as técnicas existentes na literatura, Rao [12] descreve o Método do Critério Global como uma estratégia interessante. Segundo o autor, a partir de valores alvos definidos para cada resposta de interesse, as múltiplas funções objetivo podem ser combinadas em uma única função, a qual passa a ser a função de otimização global do processo. A otimização desta função global implica na otimização dos objetivos individuais, já que a solução encontrada corresponde à combinação dos parâmetros de entrada que melhor satisfaz simultaneamente aos objetivos de todas as respostas. Sendo assim, a formulação matemática do problema abordado neste trabalho baseou-se nesta estratégia.

No entanto, tal formulação necessita que as funções objetivo entre os parâmetros de entrada e as variáveis de resposta sejam conhecidas e, para a soldagem de revestimento considerada, verifica-se que estas relações não estão definidas. Neste caso, a Metodologia de Superfície de Resposta se mostra como uma boa alternativa, na medida em que permite que as relações matemáticas entre parâmetros e respostas sejam construídas a partir de dados experimentais. Segundo Montgomery [13], a Metodologia de Superfície de Resposta é uma das técnicas do Projeto de Experimentos (DOE), definida como um conjunto de ferramentas estatísticas úteis para a modelagem e análise de problemas em que as respostas de interesse são influenciadas por múltiplos parâmetros de entrada. Vale destacar que estas ferramentas vêm sendo utilizadas com êxito na literatura para a modelagem e análise de processos de soldagem [5-9, 14, 15].

Finalmente, após a modelagem das funções objetivo e programação matemática das mesmas, empregou-se o Algoritmo Genético para a identificação do ponto de ótimo. O Algoritmo Genético, de acordo com Busacca et al. [16], é um método baseado na evolução genética natural das espécies e difere da maioria das técnicas de otimização devido ao seu critério de busca global. Assim, ao contrário dos métodos gradientes, que iniciam a busca pelo ponto de ótimo a partir de uma única solução inicial (otimização local), o Algoritmo Genético parte de uma população de soluções, sendo capaz de encontrar ótimos globais para problemas de otimização restritos e irrestritos, assim como para uma ou múltiplas funções objetivo [17].

\section{Metodologia}

Considerando o que foi apresentado anteriormente, a estratégia de otimização utilizada para o processo de revestimento do aço carbono ABNT 1020 com aço inoxidável ABNT 316L utilizando a soldagem com arame tubular foi então dividida em duas fases. Inicialmente, a Metodologia de Superfície de Resposta foi utilizada para a modelagem das funções objetivo e, para isso, envolveu as etapas de planejamento dos experimentos, procedimento experimental e modelagem matemática. Tendo definidas as funções objetivo, partiu-se para a otimização. Nesta fase, o Método do Critério Global foi utilizado para a construção da formulação do problema e a identificação do ponto de ótimo foi feita através do emprego do Algoritmo Genético.

\subsection{Planejamento dos experimentos}

Os parâmetros de soldagem analisados foram velocidade de alimentação do arame $(\mathrm{Va})$, tensão $(T)$, velocidade de soldagem $(V S)$ e distância bico de contato peça $(N)$. A partir da especificação destas variáveis, o sequenciamento dos experimentos foi então definido com base no arranjo composto central (Central Composite Design - CCD), contendo quatro parâmetros (k) em dois níveis $\left(2^{\mathrm{k}}=2^{4}=16\right)$, oito pontos axiais $(2 \mathrm{k}=8)$ e sete pontos centrais. Isto resultou em 31 experimentos. O CCD é um arranjo experimental da Metodologia de Superfície de Resposta que incorpora aos experimentos fatoriais um conjunto de pontos centrais e um conjunto de níveis extras, denominados pontos axiais [13]. Assim, permite a modelagem da influência dos 
parâmetros sobre as respostas através de equações de segunda ordem, se caracterizando como um dos arranjos mais utilizados na literatura para a análise de processos de soldagem [5-9, 14, 15]. A Figura 3 ilustra o arranjo dos experimentos para um CCD com dois parâmetros. Vale destacar que o valor adotado para $\alpha$ (distância codificada entre os pontos centrais e os pontos axiais) no presente trabalho, referente a quatro parâmetros de entrada, foi 2,0 .

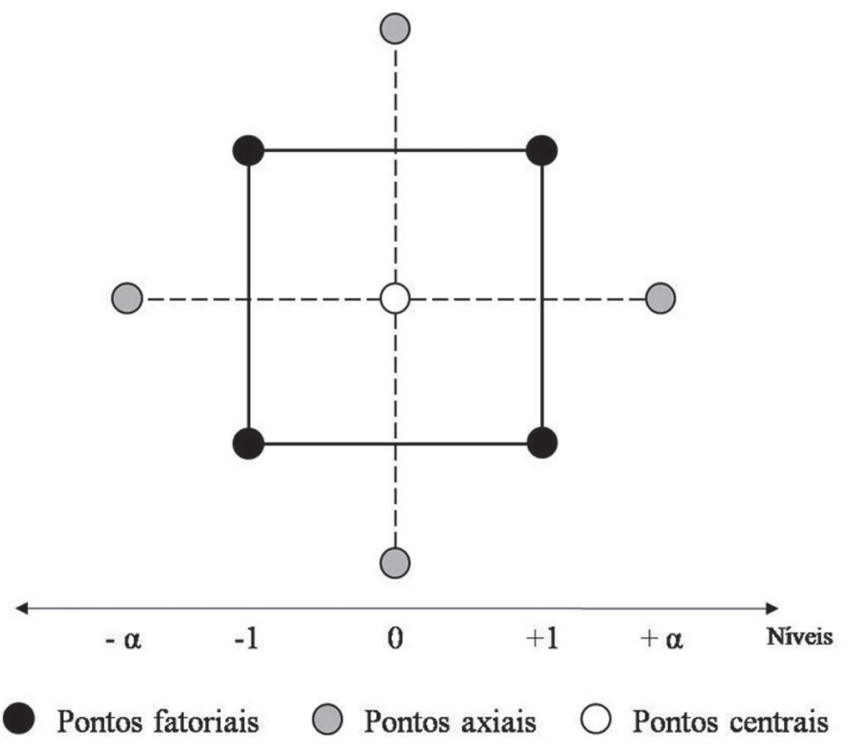

Figura 3 - Projeto composto central para dois parâmetros

Para a definição dos níveis de trabalho, foram levadas em consideração a revisão da literatura e a execução de testes preliminares. Dessa forma, pela análise dos trabalhos anteriores, os limites de cada variável foram pré-fixados de acordo com os objetivos deste trabalho. Em seguida, os níveis extremos das variáveis foram verificados através de testes preliminares para observar se o processo ocorria em tais condições. A Tabela 1 apresenta os parâmetros e seus respectivos níveis, fixados ao final dos testes preliminares.
As respostas de interesse compreendem a largura do cordão $(W)$, penetração $(P)$, reforço $(R)$ e diluição $(D)$, que representam as características geométricas do cordão de revestimento. As respostas de produtividade são a taxa de deposição (TD) e o rendimento do processo $(\eta)$. Para a qualidade, foram consideradas a formação de escória $(E)$ e o aspecto superficial $(S)$.

\subsection{Procedimento experimental}

Após a definição das variáveis de entrada, das respostas e da sequência dos experimentos, procede-se com a execução dos mesmos. Os equipamentos utilizados nesta etapa foram uma fonte ESAB AristoPower 460, um módulo AristoFeed 30-4W MA6 para a alimentação do arame e um banco de testes com dispositivo para controle da velocidade de soldagem e ajuste da tocha em relação ao metal base. $\mathrm{O}$ metal base utilizado foi o aço carbono ABNT 1020, cortado em chapas de dimensões $120 \times 60 \times 6,35 \mathrm{~mm}$. Para o metal de adição, foi empregado o arame tubular de aço inoxidável ABNT 316L, com 1,2 mm de diâmetro. A Tabela 2 apresenta a composição química dos respectivos materiais.

A execução dos experimentos foi feita através da simples deposição de um cordão de aço inoxidável sobre os corpos de prova de aço carbono, levando em consideração os parâmetros definidos na etapa de planejamento. Como gás de proteção, foi utilizado o $\mathrm{C}_{25}$ a uma vazão de $161 . \mathrm{min}^{-1}$. O ângulo da tocha foi fixado em $15^{\circ}$ na posição "empurrando".

$\mathrm{O}$ registro das respostas foi realizado na seguinte sequência: (1) avaliação das características de qualidade, (2) cálculo das respostas de produtividade e (3) medição da geometria do cordão.

Avaliação das características de qualidade: a formação de escória e o aspecto superficial foram avaliados através de notas atribuídas por especialistas envolvidos nos experimentos. Para a formação de escória, os critérios de avaliação variaram de 5 a 1 , sendo 5 a formação de escória com total recobrimento sobre o cordão e 1 a pior formação de escória, com irregularidades e defeitos. O aspecto superficial dos cordões foi avaliado

Tabela 1 - Parâmetros e seus níveis

\begin{tabular}{cccccccc}
\hline \multirow{2}{*}{ Parâmetros do processo } & \multirow{2}{*}{ Unidade } & Notação & \multicolumn{5}{c}{ Níveis dos parâmetros } \\
\cline { 5 - 9 } & & & $\mathbf{- 2}$ & $\mathbf{- 1}$ & $\mathbf{0}$ & $\mathbf{+ 1}$ & $+\mathbf{2}$ \\
\hline Velocidade de alimentação do arame & $\mathrm{m} \cdot \mathrm{min}^{-1}$ & $V a$ & 5,5 & 7,0 & 8,5 & 10,0 & 11,5 \\
Tensão & $\mathrm{V}$ & $T$ & 24,5 & 27,0 & 29,5 & 32,0 & 34,5 \\
Velocidade de soldagem & $\mathrm{cm} \cdot \mathrm{min}^{-1}$ & $V S$ & 20 & 30 & 40 & 50 & 60 \\
Distância bico de contato peça & $\mathrm{mm}$ & $N$ & 10 & 15 & 20 & 25 & 30 \\
\hline
\end{tabular}

Tabela 2 - Composição química do metal base e metal de adição

\begin{tabular}{ccccccccc}
\hline Material & $\mathbf{C}$ & $\mathbf{M n}$ & $\mathbf{P}$ & $\mathbf{S}$ & $\mathbf{S i}$ & $\mathbf{N i}$ & $\mathbf{C r}$ & $\mathbf{M o}$ \\
\hline Aço inoxidável ABNT 316L & 0,03 & 1,58 & - & - & 1,00 & 12,4 & 18,5 & 2,46 \\
Aço carbono ABNT 1020 & $0,18 / 0,23$ & $0,30 / 0,60$ & 0,04 & 0,05 & - & - & - & - \\
\hline
\end{tabular}


após a remoção da escória. Para esta avaliação, devido a uma maior quantidade de critérios adotados, as notas tiveram uma amplitude de 10 a 1 , sendo 10 a nota atribuída a um cordão isento de defeitos e com aspecto superficial liso e 1 a nota atribuída a um cordão totalmente defeituoso. A Figura 4 e a Figura 5 apresentam alguns exemplos da avaliação das características de qualidade dos cordões de revestimentos.
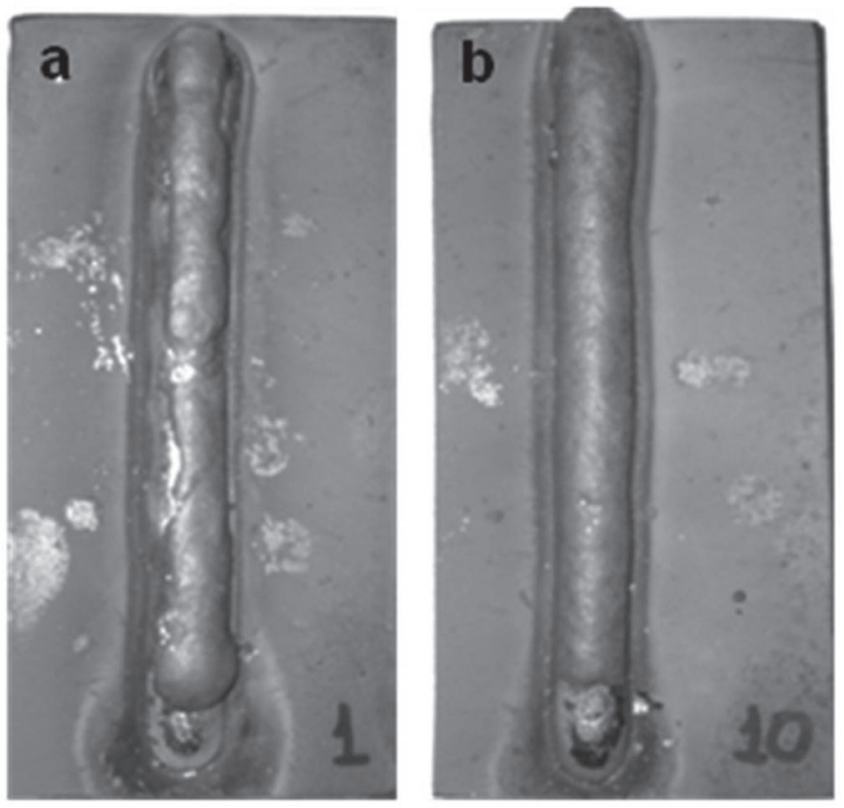

Figura 4 - Avaliação da formação de escória:

(a) Nota 3; (b) Nota 5
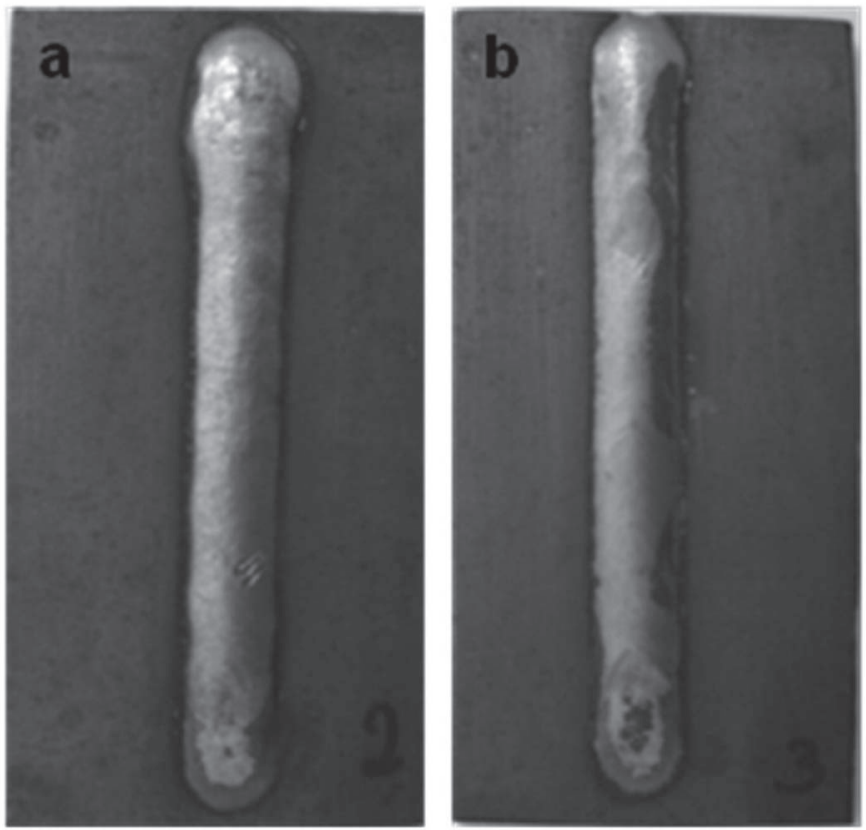

Figura 5 - Avaliação do aspecto superficial:

(a) Nota 6; (b) Nota 10

Cálculo das respostas de produtividade: para a medição das variáveis de produtividade, as chapas de aço carbono foram pesadas antes a após a deposição dos cordões e o tempo de soldagem foi cronometrado. Com isso, a taxa de fusão, a taxa de deposição e o rendimento do processo foram calculados através das seguintes expressões:

$$
\text { Taxa de fusão: } \quad T F=\frac{l_{a} \cdot d_{a} \cdot 3,6}{t_{s}} \quad\left[\mathrm{~kg} \cdot \mathrm{h}^{-1}\right]
$$

com: $\quad l_{a}-$ Comprimento do arame consumido, calculado

$$
\text { por: } l_{a}=\frac{V_{a} \cdot t_{s}}{60}[\mathrm{~m}]
$$

$V_{a}$ - Velocidade de alimentação do arame [m.min $\left.{ }^{-1}\right]$

$d_{a}^{a}$ - Densidade linear do arame: $7,21 \mathrm{~g} \cdot \mathrm{m}^{-1}$

$t_{s}$ - Tempo de soldagem $[\mathrm{s}]$

Taxa de deposição: $T D=\frac{\left(m_{f}-m_{i}\right) \cdot 3,6}{t_{s}}\left[\mathrm{~kg} \cdot \mathrm{h}^{-1}\right]$

com: $m_{i}$ - Massa da chapa antes da soldagem [g]

$m_{f}$ - Massa da chapa depois da soldagem [g]

$t_{s}$ - Tempo de soldagem [s]

Rendimento do processo: $\quad \eta=\frac{T D}{T F} \cdot 100 \quad[\%]$

Medição da geometria do cordão: as medições das respostas geométricas foram realizadas em quatro pontos distintos dos cordões, desprezando o início e o fim do processo, com a finalidade de obter a média das respostas. Os corpos de prova foram cortados e suas seções transversais foram devidamente preparadas, atacadas com nital $4 \%$ e fotografadas. A Figura 6 mostra as seções transversais de dois corpos de prova após os procedimentos de corte, preparação e ataque. Com a ajuda do software analisador de imagens Analysis Doc ${ }^{\circledR}$, as dimensões do cordão foram mensuradas, obtendo-se a largura, penetração, reforço, área de penetração e área total da solda. O percentual de diluição foi então calculado dividindo-se a área de penetração pela área total.

Após a medição de todas as respostas de interesse, estas foram reunidas para compor a matriz experimental (Tabela 3), utilizada como fonte de dados para a estimação dos modelos matemáticos. Nota-se que dois dados referentes ao reforço do cordão (testes 10 e 21) e outro referente ao aspecto superficial (teste 2) foram eliminados do estudo. Tais dados foram caracterizados como outliers e a presença dos mesmos poderia influenciar de maneira negativa a modelagem matemática das respostas.

\section{Resultados}

\subsection{Modelagem matemática das funções objetivo}

De acordo com Montgomery [13], a função polinomial de segunda ordem desenvolvida para uma superfície de resposta que relaciona uma dada resposta $y$ com $k$ variáveis de entrada apresenta o seguinte formato descrito pela Equação 5: 

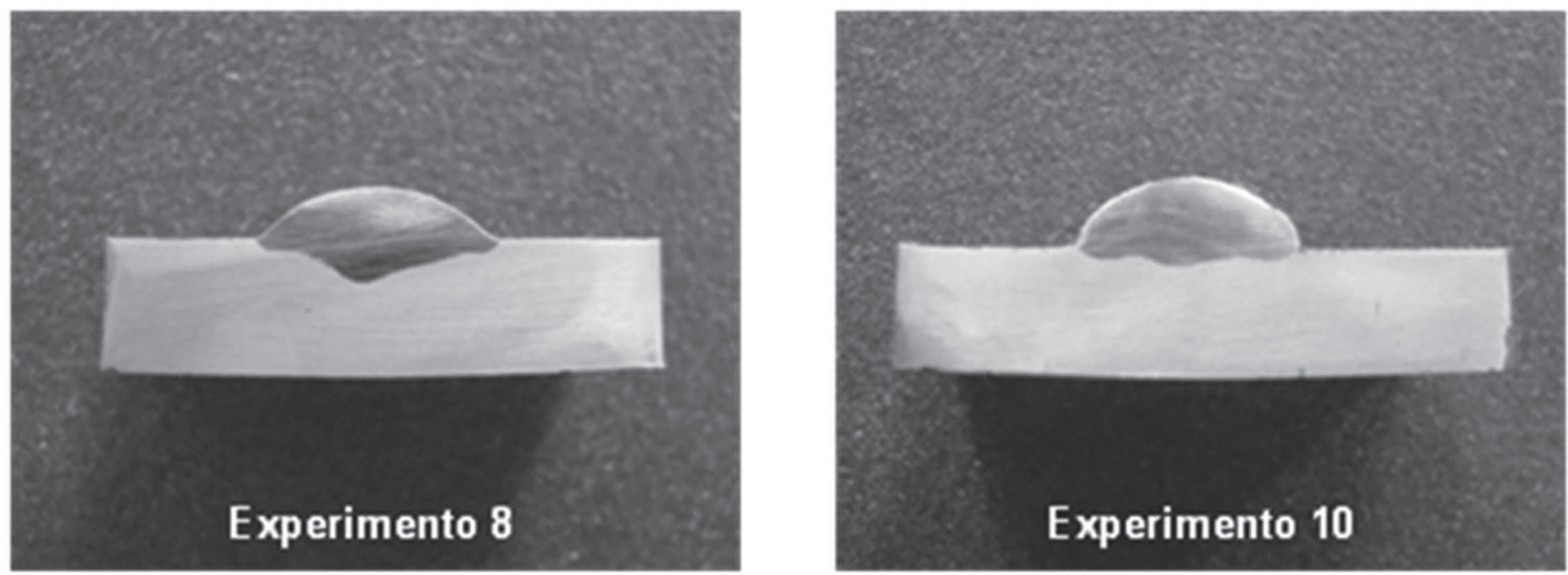

Figura 6 - Geometria dos cordões após a preparação dos corpos de prova

$y=\beta_{0}+\sum_{i=1}^{k} \beta_{i} x_{i}+\sum_{i=1}^{k} \beta_{i i} x_{i}^{2}+\sum_{i<j} \sum_{i j} x_{i} x_{j}$

sendo: $y$-Resposta de interesse

$x_{i}$ - Parâmetros de entrada

$\beta_{0}, \beta_{i}, \beta_{i i}, \beta_{i j}$ - Coeficientes a serem estimados

$k$ - Número de parâmetros de entrada considerados

Considerando o processo abordado pelo presente trabalho, em que são estudados os efeitos de quatro parâmetros, a Equação 5 pode ser escrita conforme indica a Equação 6 a seguir:

$y=\beta_{0}+\beta V a+\beta_{2} T+\beta_{3} V s+\beta_{4} N+\beta_{11} V a^{2}+\beta_{22} T^{2}+\beta_{33} V s^{2}+\beta_{44} N^{2}$

$+\beta_{12} V a T+\beta_{13} V a V s+\beta_{14} V a N+\beta_{23} T V S+\beta_{24} T N+\beta_{34} V S N$

Para o desenvolvimento dos modelos matemáticos, foi utilizado o software estatístico MINITAB ${ }^{\circledR}$, que utiliza o método dos mínimos quadrados ordinários para a obtenção dos coeficientes relacionados pelo modelo. A Tabela 4 apresenta os coeficientes estimados para os modelos quadráticos completos desenvolvidos para as respostas consideradas neste trabalho.

Após a estimação dos coeficientes, torna-se necessário verificar se os modelos desenvolvidos são adequados. Assim, o teste de adequação foi realizado através da Análise de Variância (ANOVA), feita também pelo software MINITAB ${ }^{\circledR}$. A Tabela 5 apresenta os resultados desta análise e mostra que todos os modelos desenvolvidos são adequados, pois apresentam $p$-values inferiores a 5\% de significância. Os resultados da ANOVA também indicam que, com exceção do aspecto superficial, todos os modelos desenvolvidos apresentaram bons ajustes, já que os valores de $R^{2}$ adj. foram superiores a $80 \%$. Para o aspecto superficial, o ajuste obtido foi de $61,82 \%$, considerado um ajuste não muito bom, porém aceitável.

Verificada a adequação, procede-se com a redução dos modelos através da remoção dos termos não significativos. $\mathrm{O}$ critério adotado para a remoção dos termos não significativos foi o aumento do valor de $R^{2}$ adj. e a redução do desvio-padrão $S$ dos modelos. Assim, os modelos finais apresentaram os formatos descritos pelas Equações 7 - 14 .

$\boldsymbol{W}=10,640+0,797 \mathrm{Va}+0,656 T-1,451 V s-0,629 N+0,270 V s^{2}$

$+0,26 \mathrm{VaT}-0,114 \mathrm{VaVs}-0,102 \mathrm{TV} s+0,067 V_{S} N$

$\boldsymbol{P}=1,639+0,122 \mathrm{Va}+0,122 \mathrm{~T}+0,093 \mathrm{Vs}-0,241 \mathrm{~N}+0,025 \mathrm{Va}^{2}-$

$0,032 T^{2}-0,118 \mathrm{Vs}^{2}+0,034 \mathrm{VaT}+0,076 \mathrm{VaVs}-0,100 \mathrm{VaN}$

$\boldsymbol{R}=2,597+0,191 \mathrm{Va}-0,104 \mathrm{~T}-0,223 \mathrm{VS}+0,115 \mathrm{~N}+0,034 \mathrm{~T}^{2}+$

$0,019 V s^{2}+0,036 N^{2}-0,030 V a T-0,023 V a N$

$\boldsymbol{D}=0,310-0,003 \mathrm{Va}+0,025 \mathrm{~T}+0,037 \mathrm{Vs}-0,043 \mathrm{~N}-0,007 T^{2}-$

$0,012 V^{2}+0,008 V a T+0,005 V a V s-0,004 V a N-0,008 V s N$

$\boldsymbol{T D}=3,396+0,568 \mathrm{Va}-0,009 \mathrm{~T}+0,021 \mathrm{VS}+0,031 \mathrm{~N}-0,019 \mathrm{Va}^{2}-$ $0,022 T^{2}-0,008 V^{2}-0,023 N^{2}+0,008 V a T-0,006 \mathrm{VaVs}-0,012 \mathrm{VaN}$ $-0,010 T V s+0,020 T N+0,019 V s N$

$\eta=0,924-0,006 \mathrm{Va}-0,003 \mathrm{~T}+0,006 \mathrm{Vs}+0,009 \mathrm{~N}-0,004 \mathrm{Va}^{2}-$ $0,006 T^{2}-0,002 \mathrm{Vs}^{2}-0,006 \mathrm{~N}^{2}+0,003 \mathrm{VaT}-0,003 \mathrm{VaVs}-0,005 \mathrm{VaN}$ $-0,003 T V s+0,006 T N+0,006 V s N$

$\boldsymbol{E}=3,021+0,333 \mathrm{Va}-0,333 \mathrm{~T}-0,250 \mathrm{Vs}+0,083 \mathrm{~N}+0,144 \mathrm{Va}^{2}$

$+0,144 T^{2}+0,144 V^{2}-0,250 \mathrm{VaT}-0,125 \mathrm{VaVs}+0,125 \mathrm{TVs}-$ $0,125 V s N$

$\boldsymbol{S}=7,644-0,855 \mathrm{Va}-0,272 \mathrm{~T}+0,689 \mathrm{Vs}-0,145 \mathrm{~N}+0,219 T^{2}+$ $0,219 V^{2}-0,533 \mathrm{VaT}-0,592 T N$

\subsection{Formulação do problema}

As variáveis de decisão são caracterizadas pelos parâmetros do processo, ou seja, velocidade de alimentação do arame, tensão, velocidade de soldagem e distância bico de contato peça. As funções objetivo são definidas pelos modelos matemáticos das respostas cujo valor se deseja maximizar ou minimizar. Compreendem, portanto, a largura do cordão, penetração, 
Tabela 3 - Matriz experimental

\begin{tabular}{|c|c|c|c|c|c|c|c|c|c|c|c|c|}
\hline \multirow{2}{*}{ Teste } & \multicolumn{4}{|c|}{ Parâmetros codificados } & \multicolumn{4}{|c|}{ Geometria } & \multicolumn{2}{|c|}{ Produtividade } & \multicolumn{2}{|c|}{ Qualidade } \\
\hline & $V a$ & $T$ & $\boldsymbol{V} \boldsymbol{s}$ & $N$ & $W$ & $P$ & $R$ & $D$ & $T D$ & $\eta$ & $\boldsymbol{E}$ & $S$ \\
\hline 1 & -1 & -1 & -1 & -1 & 11,19 & 1,37 & 2,63 & $26,44 \%$ & 2,718 & $89,74 \%$ & 3 & 7 \\
\hline 2 & 1 & -1 & -1 & -1 & 12,99 & 1,66 & 3,12 & $25,82 \%$ & 3,881 & $89,71 \%$ & 5 & 6 \\
\hline 3 & -1 & 1 & -1 & -1 & 12,70 & 1,69 & 2,50 & $31,49 \%$ & 2,699 & $89,14 \%$ & 3 & 10 \\
\hline 4 & 1 & 1 & -1 & -1 & 15,05 & 1,98 & 2,78 & $31,25 \%$ & 3,871 & $89,47 \%$ & 3 & $*$ \\
\hline 5 & -1 & -1 & 1 & -1 & 9,21 & 1,65 & 2,17 & $36,22 \%$ & 2,773 & $91,58 \%$ & 3 & 10 \\
\hline 6 & 1 & -1 & 1 & -1 & 9,96 & 1,94 & 2,67 & $33,69 \%$ & 3,924 & $90,70 \%$ & 4 & 9 \\
\hline 7 & -1 & 1 & 1 & -1 & 9,75 & 1,54 & 2,06 & $37,12 \%$ & 2,647 & $87,43 \%$ & 3 & 10 \\
\hline 8 & 1 & 1 & 1 & -1 & 11,51 & 2,18 & 2,42 & $41,08 \%$ & 3,822 & $88,36 \%$ & 3 & 8 \\
\hline 9 & -1 & -1 & -1 & 1 & 10,32 & 1,25 & 2,87 & $22,46 \%$ & 2,740 & $90,49 \%$ & 4 & 9 \\
\hline 10 & 1 & -1 & -1 & 1 & 11,43 & 1,00 & $*$ & $18,32 \%$ & 3,870 & $89,47 \%$ & 5 & 8 \\
\hline 11 & -1 & 1 & -1 & 1 & 11,27 & 1,32 & 2,85 & $23,71 \%$ & 2,743 & $90,60 \%$ & 3 & 7 \\
\hline 12 & 1 & 1 & -1 & 1 & 13,34 & 1,10 & 3,18 & $21,96 \%$ & 3,885 & $89,81 \%$ & 4 & 4 \\
\hline 13 & -1 & -1 & 1 & 1 & 7,99 & 1,11 & 2,55 & $24,96 \%$ & 2,847 & $94,03 \%$ & 3 & 9 \\
\hline 14 & 1 & -1 & 1 & 1 & 8,62 & 1,23 & 2,80 & $23,31 \%$ & 3,901 & $90,17 \%$ & 4 & 9 \\
\hline 15 & -1 & 1 & 1 & 1 & 8,48 & 1,37 & 2,36 & $28,77 \%$ & 2,832 & $93,52 \%$ & 3 & 10 \\
\hline 16 & 1 & 1 & 1 & 1 & 10,84 & 1,64 & 2,60 & $30,19 \%$ & 3,969 & $91,74 \%$ & 3 & 7 \\
\hline 17 & -2 & 0 & 0 & 0 & 9,07 & 1,38 & 2,21 & $31,56 \%$ & 2,204 & $92,62 \%$ & 3 & 9 \\
\hline 18 & 2 & 0 & 0 & 0 & 12,21 & 2,14 & 3,06 & $30,95 \%$ & 4,454 & $89,52 \%$ & 4 & 6 \\
\hline 19 & 0 & -2 & 0 & 0 & 9,42 & 1,20 & 3,03 & $22,84 \%$ & 3,324 & $90,41 \%$ & 4 & 9 \\
\hline 20 & 0 & 2 & 0 & 0 & 11,69 & 1,86 & 2,46 & $35,58 \%$ & 3,311 & $90,04 \%$ & 3 & 8 \\
\hline 21 & 0 & 0 & -2 & 0 & 14,93 & 0,95 & $*$ & $18,58 \%$ & 3,319 & $90,27 \%$ & 4 & 8 \\
\hline 22 & 0 & 0 & 2 & 0 & 8,48 & 1,43 & 2,25 & $35,78 \%$ & 3,423 & $93,08 \%$ & 3 & 9 \\
\hline 23 & 0 & 0 & 0 & -2 & 11,73 & 2,18 & 2,61 & $40,44 \%$ & 3,242 & $88,15 \%$ & 3 & 8 \\
\hline 24 & 0 & 0 & 0 & 2 & 9,22 & 1,28 & 2,89 & $24,16 \%$ & 3,385 & $92,05 \%$ & 3 & 8 \\
\hline 25 & 0 & 0 & 0 & 0 & 10,82 & 1,71 & 2,60 & $31,05 \%$ & 3,421 & $93,04 \%$ & 3 & 8 \\
\hline 26 & 0 & 0 & 0 & 0 & 10,93 & 1,72 & 2,59 & $31,67 \%$ & 3,380 & $91,91 \%$ & 3 & 8 \\
\hline 27 & 0 & 0 & 0 & 0 & 10,74 & 1,62 & 2,65 & $30,88 \%$ & 3,402 & $92,51 \%$ & 3 & 7 \\
\hline 28 & 0 & 0 & 0 & 0 & 10,61 & 1,80 & 2,50 & $32,83 \%$ & 3,382 & $91,98 \%$ & 3 & 8 \\
\hline 29 & 0 & 0 & 0 & 0 & 10,64 & 1,49 & 2,62 & $29,99 \%$ & 3,388 & $92,15 \%$ & 3 & 7 \\
\hline 30 & 0 & 0 & 0 & 0 & 10,59 & 1,49 & 2,61 & $31,09 \%$ & 3,398 & $92,40 \%$ & 3 & 7 \\
\hline 31 & 0 & 0 & 0 & 0 & 10,57 & 1,50 & 2,56 & $31,02 \%$ & 3,404 & $92,58 \%$ & 3 & 8 \\
\hline
\end{tabular}


Tabela 4 - Coeficientes estimados para os modelos quadráticos completos

\begin{tabular}{|c|c|c|c|c|c|c|c|c|}
\hline \multirow{2}{*}{ Coeficiente } & \multicolumn{8}{|c|}{ Respostas } \\
\hline & $W$ & $P$ & $R$ & $D$ & $T D$ & $\eta$ & $E$ & $S$ \\
\hline Constante & 10,6996 & 1,6192 & 2,5898 & 0,3122 & 3,3964 & 0,9237 & 3,0000 & 7,5714 \\
\hline$\beta_{1}$ & 0,7967 & 0,1221 & 0,1921 & $-0,0028$ & 0,5676 & $-0,0055$ & 0,3333 & $-0,8792$ \\
\hline$\beta_{2}$ & 0,6555 & 0,1220 & $-0,1051$ & 0,0249 & $-0,0088$ & $-0,0027$ & $-0,3333$ & $-0,2958$ \\
\hline$\beta_{3}$ & $-1,4507$ & 0,0934 & $-0,2230$ & 0,0368 & 0,0214 & 0,0061 & $-0,2500$ & 0,7125 \\
\hline$\beta_{4}$ & $-0,6290$ & $-0,2408$ & 0,1155 & $-0,0425$ & 0,0308 & 0,0090 & 0,0833 & $-0,1208$ \\
\hline$\beta_{11}$ & $-0,0033$ & 0,0266 & 0,0069 & $-0,0023$ & $-0,0190$ & $-0,0039$ & 0,1458 & $-0,0293$ \\
\hline$\beta_{22}$ & $-0,0240$ & $-0,0300$ & 0,0346 & $-0,0074$ & $-0,0218$ & $-0,0060$ & 0,1458 & 0,2207 \\
\hline$\beta_{33}$ & 0,2637 & $-0,1161$ & 0,0196 & $-0,0125$ & $-0,0084$ & $-0,0024$ & 0,1458 & 0,2207 \\
\hline$\beta_{44}$ & $-0,0440$ & 0,0190 & 0,0368 & 0,0003 & $-0,0229$ & $-0,0063$ & 0,0208 & 0,0957 \\
\hline$\beta_{12}$ & 0,2663 & 0,0337 & $-0,0309$ & 0,0077 & 0,0080 & 0,0028 & $-0,2500$ & $-0,5688$ \\
\hline$\beta_{13}$ & $-0,1137$ & 0,0757 & $-0,0146$ & 0,0050 & $-0,0057$ & $-0,0026$ & $-0,1250$ & 0,1938 \\
\hline$\beta_{14}$ & $-0,0308$ & $-0,0998$ & $-0,0219$ & $-0,0042$ & $-0,0124$ & $-0,0049$ & 0,0000 & 0,0687 \\
\hline$\beta_{23}$ & $-0,1023$ & 0,0002 & $-0,0049$ & 0,0023 & $-0,0103$ & $-0,0032$ & 0,1250 & 0,0687 \\
\hline$\beta_{24}$ & $-0,0064$ & 0,0048 & 0,0148 & $-0,0020$ & 0,0204 & 0,0055 & 0,0000 & $-0,5563$ \\
\hline$\beta_{34}$ & 0,0665 & 0,0045 & $-0,0144$ & $-0,0077$ & 0,0195 & 0,0057 & $-0,1250$ & $-0,0688$ \\
\hline
\end{tabular}

Coeficientes em negrito indicam os termos significativos

Tabela 5 - Análise de Variância

\begin{tabular}{|c|c|c|c|c|c|c|c|c|c|}
\hline \multirow{2}{*}{ Resposta } & \multicolumn{2}{|c|}{ Graus de liberdade } & \multicolumn{2}{|c|}{ Soma dos quadrados } & \multicolumn{2}{|c|}{ Média quadrática } & \multirow{2}{*}{$F$} & \multirow{2}{*}{ p-value } & \multirow{2}{*}{$\begin{array}{c}R^{2} \text { adj } \\
(\%)\end{array}$} \\
\hline & Regressão & Residual & Regressão & Residual & Regressão & Residual & & & \\
\hline$W$ & 14 & 16 & 89,355 & 0,975 & 6,383 & 0,061 & 104,73 & 0,000 & 97,98 \\
\hline$P$ & 14 & 16 & 3,054 & 0,300 & 0,218 & 0,019 & 11,64 & 0,000 & 83,24 \\
\hline$R$ & 14 & 14 & 2,067 & 0,088 & 0,148 & 0,006 & 23,58 & 0,000 & 91,86 \\
\hline$D$ & 14 & 16 & 0,099 & 0,004 & 0,007 & 0,000 & 31,49 & 0,000 & 93,43 \\
\hline$T D$ & 14 & 16 & 7,817 & 0,008 & 0,558 & 0,000 & 1131,65 & 0,000 & 99,81 \\
\hline$\eta$ & 14 & 16 & 0,008 & 0,001 & 0,001 & 0,000 & 12,92 & 0,000 & 84,77 \\
\hline$E$ & 14 & 16 & 10,263 & 0,833 & 0,733 & 0,052 & 14,08 & 0,000 & 85,92 \\
\hline$S$ & 14 & 15 & 42,507 & 10,460 & 3,036 & 0,697 & 4,35 & 0,004 & 61,82 \\
\hline
\end{tabular}

Valores tabulados da distribuição de Fisher: $F_{95 \%}(14,16)=2.37 ; F_{95 \%}(14,15)=2.42 ; F_{95 \%}(14,14)=2.48$ 
reforço, diluição, taxa de deposição e rendimento do processo. A formação de escória e o aspecto superficial foram tratados como restrições, já que se deseja que o processo seja otimizado sem a ocorrência de defeitos e com pequenas falhas na formação da escória.

Considerando que o problema em questão emprega a otimização de múltiplos objetivos, foi escolhido o Método do Critério Global como técnica de programação matemática. Segundo Rao [12], a partir de valores alvos definidos para cada objetivo, o problema pode ser formulado como a minimização da soma dos quadrados dos desvios relativos das funções objetivo individuais em relação aos valores alvo, ou seja,

$$
\begin{aligned}
\operatorname{Min} F(\mathrm{X}) & =\sum_{i=1}^{k}\left\{\frac{T_{i}-f_{i}(\mathrm{X})}{T_{i}}\right\}^{2} \\
& \text { Sujeito } a: \quad g_{j}(\mathrm{X}) \leq 0, \quad j=1,2, \ldots, m
\end{aligned}
$$

onde: $F(\mathrm{X})$ - Critério Global

$T_{i}$ - Alvo definido para o i-ésimo objetivo

$f_{i}(\mathrm{X})$ - Função do i-ésimo objetivo

$g_{j}(\mathrm{X})-$ Restrições

$k$ - Número de objetivos

Assim, definidos os valores alvo para cada resposta de interesse, os múltiplos objetivos são combinados em uma única função, que passa a ser a função de otimização global do processo. A determinação dos alvos das respostas de interesse foi feita através da minimização / maximização individual dos modelos representados pelas Equações 7 - 14, levando em consideração os seguintes objetivos:

- Maximização da largura do cordão

- Minimização da penetração

- Maximização do reforço

- Minimização da diluição

- Maximização da taxa de deposição

- Maximização do rendimento
Com isso, chegou-se à formulação final do problema, expressa por:

$$
\begin{aligned}
& \text { Min } G=\left(\frac{15,57-W}{15,57}\right)^{2}+\left(\frac{0,83-P}{0,83}\right)^{2}+\left(\frac{3,34-R}{3,34}\right)^{2} \\
& +\left(\frac{16,27-D}{16,27}\right)^{2}+\left(\frac{4,46-T D}{4,46}\right)^{2}+\left(\frac{94,90-\eta}{94,90}\right)^{2}
\end{aligned}
$$

Sujeito a: $\quad E \geq 4$

$$
S \leq 8
$$$$
V a, T, V s, N \geq-2
$$$$
\text { Va, } T, V s, N \leq+2
$$

onde: $G$ - Critério Global

$W, P, R, D, T D, \eta, E, S$ - Modelos de superfície de resposta definidos pelas Equações 7 - 14

$V a, T, V s, N-$ Variáveis de decisão nos seus valores codificados

\subsection{Identificação do ponto de ótimo}

Para a identificação do ponto de ótimo, foi construída uma planilha no Microsoft Excel®, onde foi feita a programação matemática da formulação anterior. O Algoritmo Genético foi utilizado como algoritmo de otimização, através do suplemento Solver Evolutionary do software. Optou-se pelo emprego do Algoritmo Genético em função do mesmo ser vantajoso quando se deseja a otimização global do processo. Finalmente, após executar o algoritmo cinco vezes partindo de condições iniciais distintas e aleatórias, o ponto de ótimo foi determinado. A Tabela 6 descreve os parâmetros definidos para o uso do Algoritmo Genético e a Tabela 7 apresenta os resultados otimizados do processo de revestimento, obtidos com um nível de significância de $5 \%$ e com os seguintes parâmetros em sua forma decodificada: $V a=10,2 \mathrm{~m} \cdot \mathrm{min}^{-1} ; T=26,1 \mathrm{~V} ; V_{S}=25,4 \mathrm{~cm} \cdot \mathrm{min}^{-1} ; N=22.1 \mathrm{~mm}$.

\begin{tabular}{|c|c|c|c|c|c|c|c|c|}
\hline \multirow{2}{*}{ Respostas } & \multicolumn{4}{|c|}{ Geometria } & \multicolumn{2}{|c|}{ Produtividade } & \multicolumn{2}{|c|}{ Qualidade } \\
\hline & $W$ & $P$ & $R$ & $D$ & $T D$ & $\eta$ & $E$ & $S$ \\
\hline Valores ótimos & $12,63 \pm 0,45$ & $0,87 \pm 0,22$ & $3,48 \pm 0,16$ & $14,43 \pm 2,52$ & $3,91 \pm 0,05$ & $87,78 \pm 1,41$ & 5 & 8 \\
\hline Alvos & 15,57 & 0,83 & 3,34 & 16,27 & 4,46 & 94,90 & $\geq 4$ & $\geq 8$ \\
\hline Unidade & $\mathrm{mm}$ & $\mathrm{mm}$ & $\mathrm{mm}$ & $\%$ & $\mathrm{~kg} \cdot \mathrm{h}^{-1}$ & $\%$ & nota & nota \\
\hline
\end{tabular}

Tabela 6 - Parâmetros definidos para o Algoritmo Genético

\begin{tabular}{cc}
\hline Parâmetros & Valores \\
\hline Número de iterações & 1.000 \\
Tamanho da população & 150 \\
Taxa de mutação & $5 \%$ \\
Convergência & 0,0001 \\
\hline
\end{tabular}

Tabela 7 - Resultados ótimos para a soldagem de revestimento do aço carbono ABNT 1020 com arame tubular de aço inoxidável ABNT 316L 


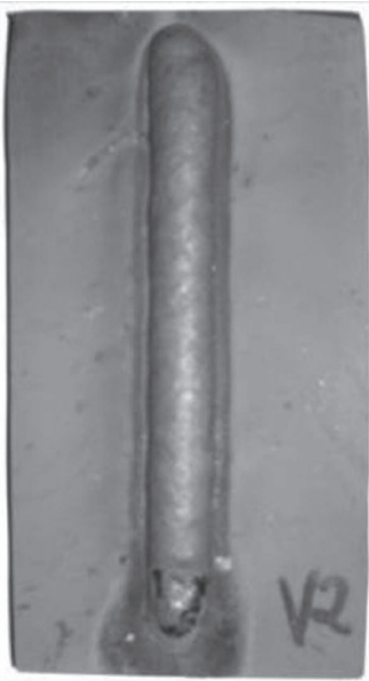

Figura 7 - Formação de escória ótima

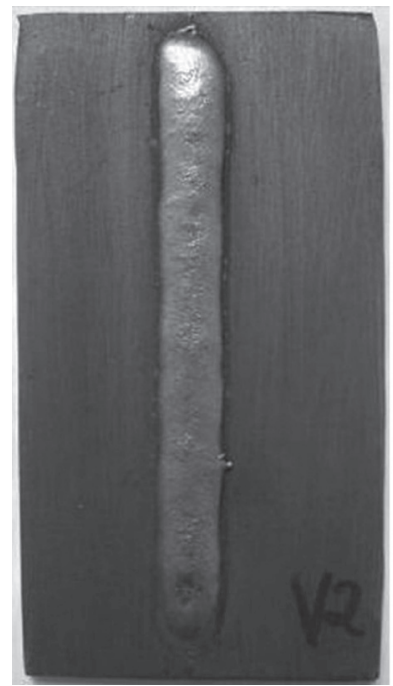

Figura 8 - Aspecto superficial ótimo
As Figuras 7 - 9 apresentam respectivamente a formação de escória ótima, o aspecto superficial ótimo e a geometria ótima. Observa-se que o perfil geométrico obtido condiz com o perfil desejado, o que caracteriza o ponto ótimo identificado como um resultado satisfatório.

A Tabela 7 também apresenta uma comparação entre os resultados ótimos e os alvos definidos para cada resposta. Observa-se que as características geométricas, com exceção da largura do cordão, apresentaram os valores ótimos próximos aos valores alvo, já que estes últimos se encontram inseridos dentro do intervalo de confiança de $95 \%$ obtido para as respostas ótimas. Além disso, os resultados para o reforço e diluição superaram os objetivos definidos. Quanto à largura do cordão, em comparação com as outras respostas, observa-se um maior desvio do valor ótimo em relação ao alvo, estando este fora do intervalo de confiança. No entanto, tal resultado foi considerado satisfatório devido ao bom perfil geométrico apresentado pela Figura 9. As respostas de produtividade também apresentaram os intervalos de confiança fora dos alvos, com resultados ótimos um pouco abaixo do especificado. Mesmo assim, tais resultados foram considerados satisfatórios, dado que a taxa de deposição ficou próximo a $4 \mathrm{~kg} \cdot \mathrm{h}^{-1}$ e o rendimento próximo a $90 \%$. Para a qualidade superficial, verifica-se que o processo foi otimizado na condição mínima exigida para a formação de escória e para o aspecto superficial, sendo atendidas ambas as restrições.

Apesar dos resultados anteriores serem caracterizados como satisfatórios, é conveniente ressaltar que os mesmos foram obtidos empregando-se uma única estratégia de otimização. Além disso, as respostas otimizadas foram tratadas com o mesmo grau de importância e a estrutura de correlação entre elas não foi levada em consideração. Dessa forma, a utilização de outras abordagens empregando programações matemáticas diferentes, algoritmos diferentes, pesos diferentes entre as respostas ou considerando a correlação entre elas, pode conduzir os resultados a pontos de ótimo diferentes.

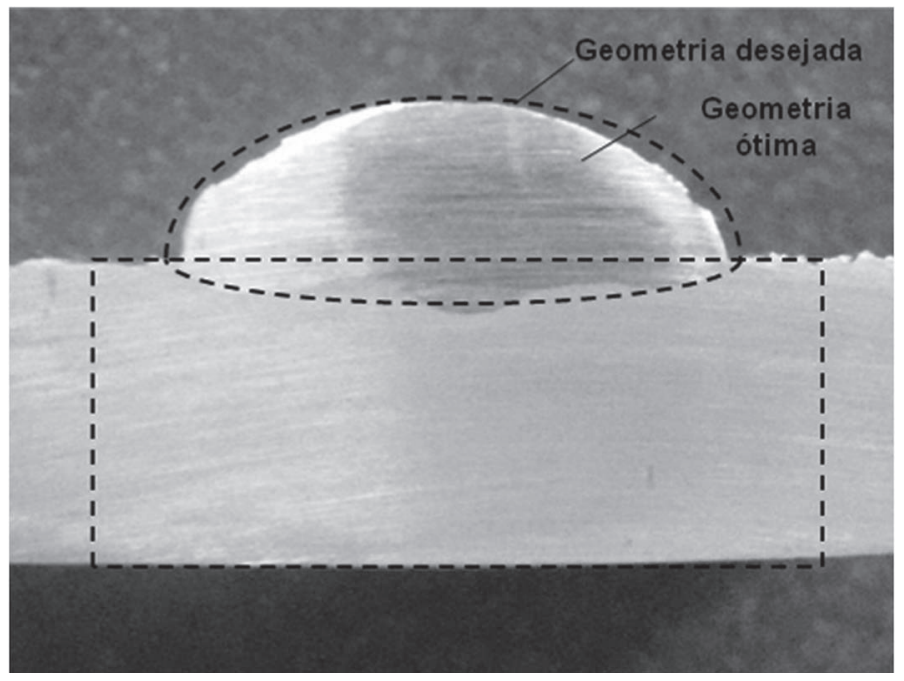

Figura 9-Geometria ótima do cordão de revestimento

\section{Conclusões}

A partir dos resultados anteriores, estabelecem-se as seguintes conclusões:

1. A aplicação dos métodos de otimização aos processos de soldagem requer que as funções entre os parâmetros do processo e as respostas de interesse sejam conhecidas. Para a determinação de tais relações matemáticas, técnicas experimentais, como a Metodologia de Superfície de Resposta, podem ser eficientemente empregadas.

2. Os modelos de superfície de resposta desenvolvidos foram caracterizados como expressões de grande confiabilidade, já que apresentaram altos ajustes. Com exceção do aspecto superficial, todos os modelos apresentaram ajustes superiores a $80 \%$. Embora o ajuste obtido para o aspecto superficial tenha sido inferior a $80 \%$, este foi considerado satisfatório.

3. A combinação do Método do Critério Global com o Algoritmo Genético foi aplicada com sucesso, o que permitiu a identificação do ponto de ótimo.

4. Considerando um nível de significância de 5\% (intervalos de confiança de 95\%), a soldagem de revestimento do aço carbono ABNT 1020 com arame tubular de aço inoxidável ABNT 316L é otimizada através do emprego dos seguintes parâmetros: $V a=10,2 \mathrm{~m} \cdot \mathrm{min}^{-1} ; T=26,1 \mathrm{~V} ; V s=25,4 \mathrm{~cm} \cdot \mathrm{min}^{-1}$; $N=22,1 \mathrm{~mm}$. Tal combinação conduz às seguintes respostas ótimas: $W=12,63 \pm 0,45 \mathrm{~mm} ; P=0,87 \pm 0,22 \mathrm{~mm} ; R=3,48$ $\pm 0,16 \mathrm{~mm} ; D=14,43 \pm 2,52 \% ; T D=3,91 \pm 0,05 \mathrm{~kg} \cdot \mathrm{h}^{-1} ; \eta=$ $87,78 \pm 1,41 \% ; E=$ nota $5 ; S=$ nota 8 .

5. O perfil geométrico observado na condição otimizada do processo foi próximo ao perfil teórico desejado para a soldagem de revestimento. Apesar de um maior desvio em relação ao alvo ser observado para a largura do cordão, considerou-se tal desvio satisfatório devido ao bom perfil geométrico ótimo observado.

6. A otimização da produtividade apresentou um resultado um 
pouco abaixo do desejado. No entanto, tais resultados também foram considerados satisfatórios. A taxa de deposição ótima apresentou valores próximos a $4 \mathrm{~kg} \cdot \mathrm{h}^{-1}$ e o rendimento foi próximo a $90 \%$.

7. Como consideração para estudos futuros, sugere-se a aplicação de outras estratégias de otimização a este problema, com a finalidade de comparação dos resultados.

\section{Agradecimentos}

Os autores agradecem a Capes, CNPq, FAPEMIG e o Instituto de Engenharia Mecânica da UNIFEI pelo apoio à realização deste trabalho.

\section{Referências Bibliográficas}

[1] PHILLIPS, A. L. Welding Handbook: Special Welding Processes and Cutting. London: American Welding Society, 1965b. 4. Vol. 3.

[2] MARQUES, P. V.; MODENESI, P. J.; BRACARENSE, A. Q. Soldagem: fundamentos e tecnologia. Belo Horizonte: UFMG, 2005. $362 \mathrm{p}$.

[3] MURUGAN, N. e PARMAR, R. S. Stainless steel cladding deposited by automatic gas metal arc welding. Welding Journal, v. 76, p. 391s-403s, 1997.

[4] MURUGAN, N e PARMAR, R. S. Effects of MIG process parameters on the geometry of the bead in the automatic surfacing of stainless steel. Journal of Materials Processing Technology, v. 41, n. 4, p. 381-398, 1994.

[5] PALANI, P. K. e MURUGAN, N. Development of mathematical models for prediction of weld bead geometry in cladding by flux cored arc welding. International Journal of Advanced Manufacturing Technology, v. 30, n. 7-8, p. 669-676, 2006.

[6] PALANI, P. K. e MURUGAN, N. Optimization of weld bead geometry for stainless steel claddings deposited by FCAW. Journal of Materials Processing Technology, v. 190, n. 1-3, p. 291-299, 2007.

[7] KANNAN, T. e MURUGAN, N. Effect of flux cored arc welding process parameters on duplex stainless steel clad quality. Journal of Materials Processing Technology, v. 176, n. 1-3, p. 230-239, 2006.

[8] SHAHI, A. S. e PANDEY, S. Prediction of dilution in GMA and UGMA stainless steel single layer cladding using response surface methodology. Science and Technology of Welding and Joining, v. 11, n. 6, p. 634-640, 2006.

[9] BALASUBRAMANIAN, V.; LAKSHMINARAYANAN, A. K.; VARAHAMOORTHY, R. e BABU, S. Application of response surface methodology to prediction of dilution in plasma transferred arc hardfacing of stainless steel on carbon steel. International Journal of Iron and Steel Research, v. 16, n. 1, p. 44-53, 2009.

[10] SHAHI, A. S. e PANDEY, S. Modelling of the effects of welding conditions on dilution of stailess steel claddings produced by gas metal arc welding procedures. Journal of Materials Processing Technology, v. 196, n. 1-3, p. 339-344, 2008 .
[11] JEFFUS, L. Welding: principles and applications. 5 ed. Australia: Delmar Learning, 2004. 904 p.

[12] RAO, S. S. Engineering optimization: theory and practice. 3ed. USA: John Wiley \& Sons, 1996. 903 p.

[13] MONTGOMERY, D. C. Design and Analysis of Experiments. 6 ed. New York: John Wiley, 2005. 643 p.

[14] RODRIGUeS, L. O.; PAIVA, A. P. e COSTA, S. C. Otimização do processo de soldagem com eletrodo tubular através da análise da geometria do cordão de solda. Soldagem \& Inspeção, v. 13, n. 2, p. 118-127, 2008.

[15] PAIVA, E. J.; RODRIGUES, L. O.; COSTA, S. C.; PAIVA, A. P. e BALESTRASSI, P. P. Otimização do processo de soldagem FCAW usando o Erro Quadrático Médio Multivariado. Soldagem \& Inspeção, v. 15, n. 1, p. 31-40, 2010.

[16] BUSACCA, G. P.; MARSEGUERRA, M. e ZIO, E. Multiobjective optimization by Genetic Algorithms: Application to safety systems. Reliability Engineering \& System Safety, v. 72, n. 1, p. 59-74, 2001.

[17] ZAIN, A. M.; HARON, H. e SHARIF, S. Application of GA to optimize cutting conditions for minimizing surface roughness in end milling machining process. Expert Systems With Applications, v. 37, n. 6, p. 4650-4659, 2010. 